\title{
Effects of oxygen content and light intensity on milk photooxidation using untargeted metabolomics analysis
}

Dongfei Tan a , Aijin Ma ${ }^{\text {b }}$, Shaolei Wang a , Qingyang Zhang a , Man Jia a , Afaf Kamal-Eldin ${ }^{\text {c, }}$ Huaxing $\mathrm{Wu}^{\mathrm{d}, \mathrm{e}}$, Gang Chen ${ }^{\mathrm{a}}$

${ }^{\text {a }}$ Key Laboratory of Agro-Product Quality and Safety, Institute of Quality Standards and Testing Technology for Agro-Products, Chinese Academy of Agricultural Sciences (CAAS), Beijing, China, 100081

${ }^{\mathrm{b}}$ School of Food and Health, Beijing Technology and Business University, Beijing, 100048, China

${ }^{\mathrm{c}}$ College of Food and Agriculture, Department of Food, Nutrition and Health (CFA), United Arab Emirates University, Al Ain, United Arab Emirates

${ }^{\mathrm{d}}$ Baijiu Science and Research Center, Sichuan Swellfun Co., Ltd. Chengdu, China, 610036

${ }^{\text {e }}$ Dairy Nutrition and Function, Key Laboratory of Sichuan Province, New Hope Dairy Company Limited, Product Research and Development Center, Chengdu, China, 610023

tandongfei1991@163.com; maaj@btbu.edu.cn; w_shaolei2018@163.com;

857729748@qq.com; 435879722@qq.com; afaf.kamal@uaeu.ac.ae;

wuhuaxing@swellfun.com; chengang01@caas.cn

Corresponding Author :

E-mail: wuhuaxing@swellfun.com (Huaxing Wu)

Or

E-mail: chengang01@caas.cn (Gang Chen) 
A

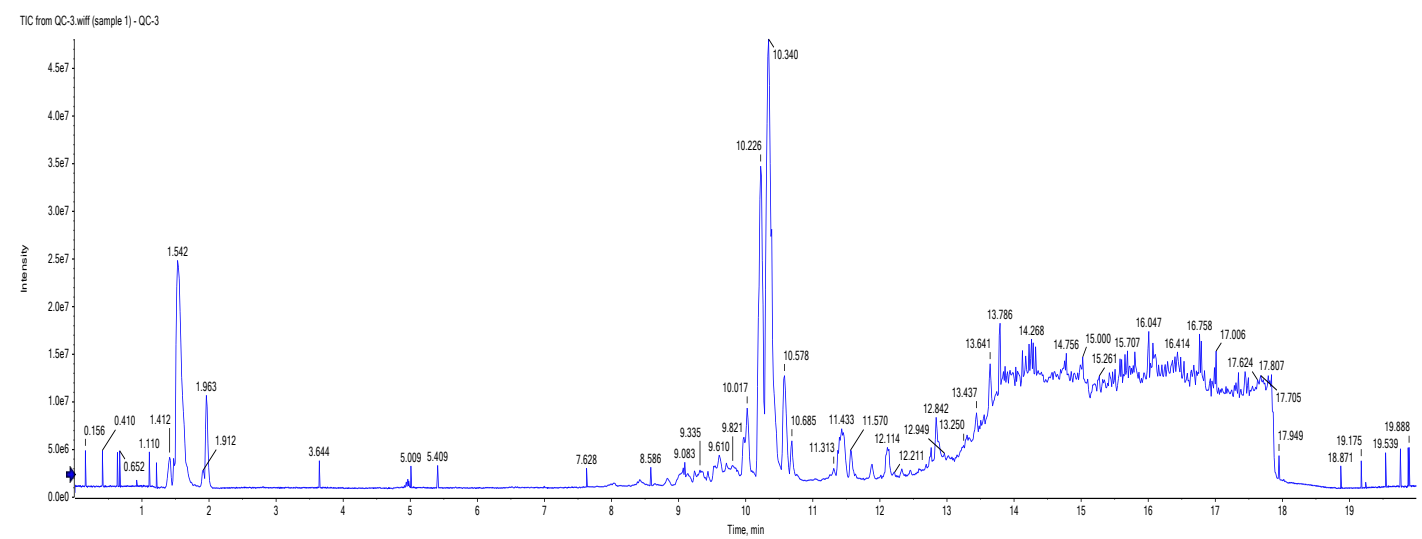

B

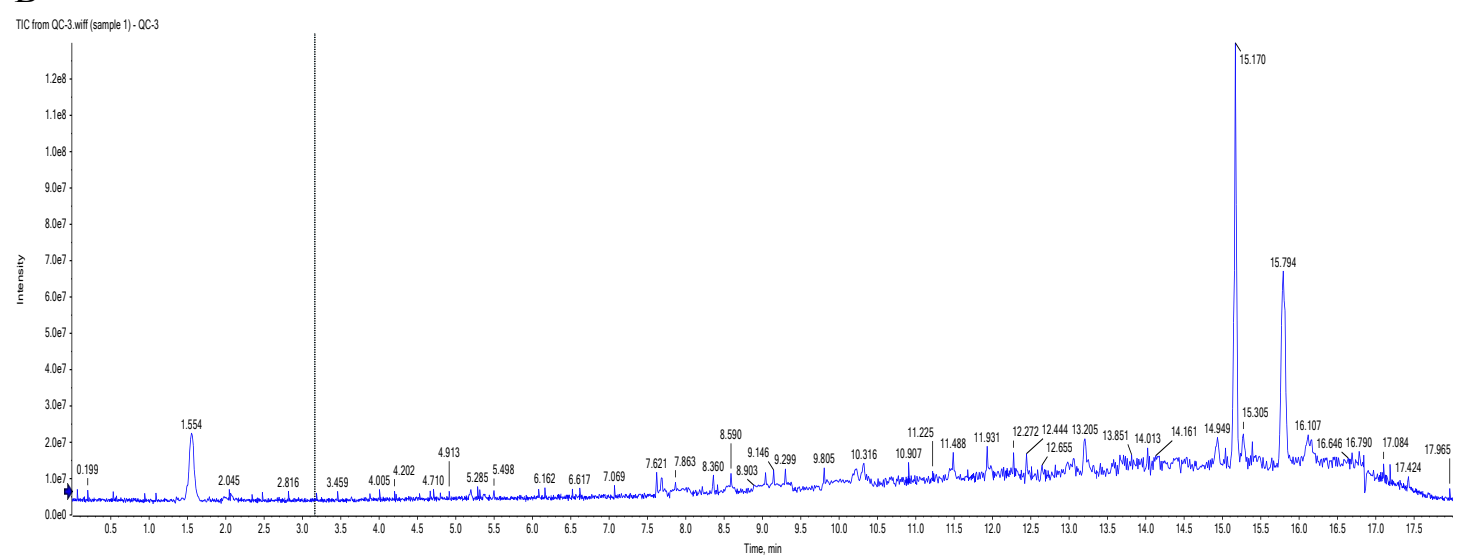

Fig S1 Total ion chromatography of QC sample in positive (A) and negative ionization mode (B) using high performance liquid chromatography-triple time-of-flight MS spectrometry

A. PCA Score Plot in POS

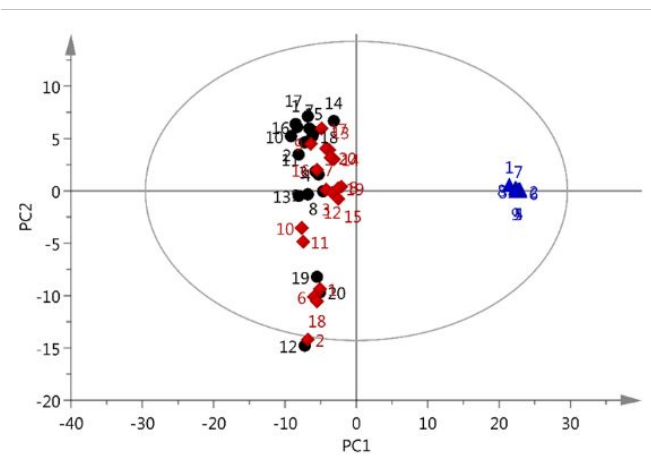

B. PCA Score Plot in NEG

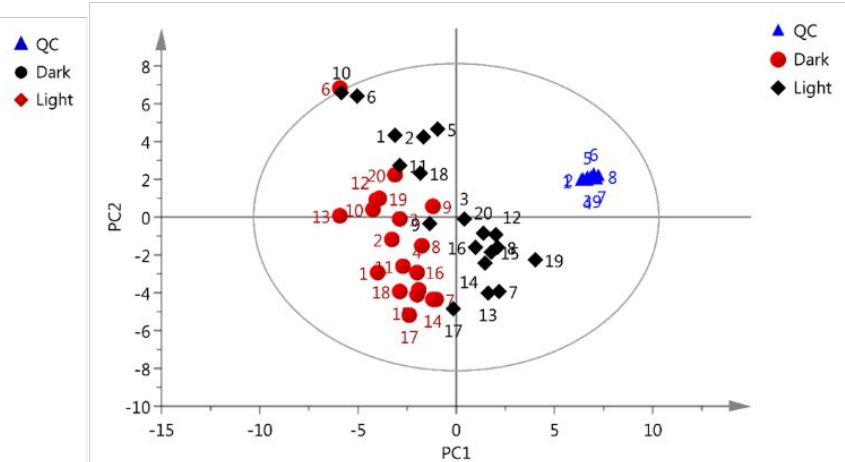

Fig S2 PCA score plot in positive (A) and negative(B) ionization mode of milk samples (QC, pooled milk; Dark, dark group milk; Light, light group milk; $\mathrm{n}=20$ ) 

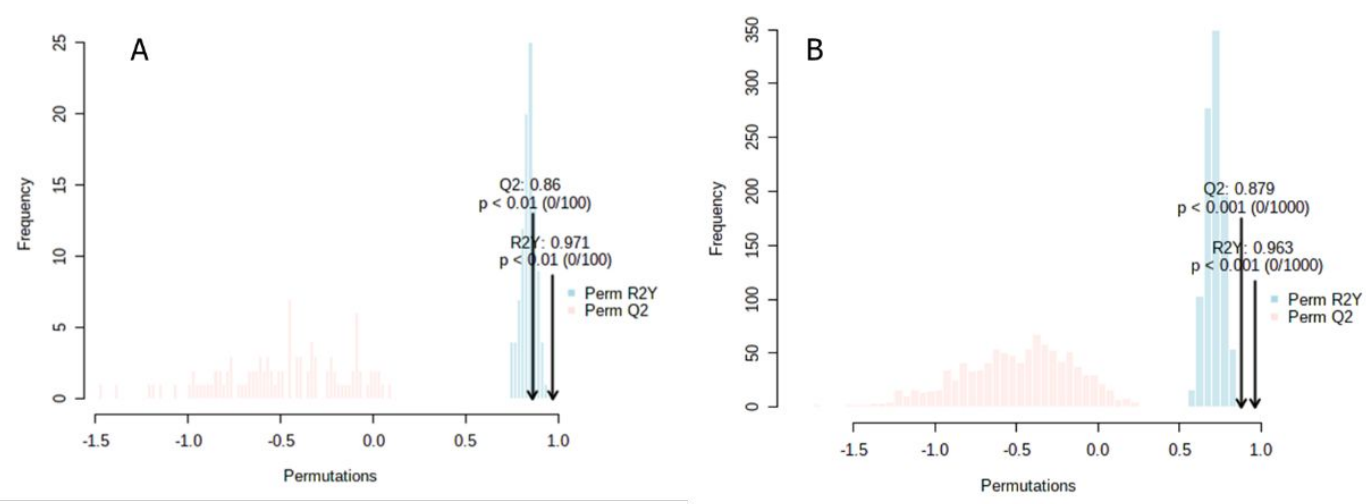

Fig S3. Permutation plot for OPLS-DA models in positive (A, Empirical p-value R2Y: $p<0.01(0 / 1000)$ and Q2: $p<0.01(0 / 1000))$ and negative $(B$, Empirical p-value R2Y: $p<0.001(0 / 1000)$ and Q2: $p<$ $0.001(0 / 1000))$ ionization mode

A

- Spectrum from QC-11. wiff (sample 1) - QC-11, Experiment 3, +TOF MS^2 (50 - 1000) from $10.752 \mathrm{~min}$

Precursor: $146.1 \mathrm{Da}, \mathrm{CE}: 35.0 \mathrm{CE}=35$
O Library Spectrum: IAld $(487-89-8), C E=35 \pm 15$

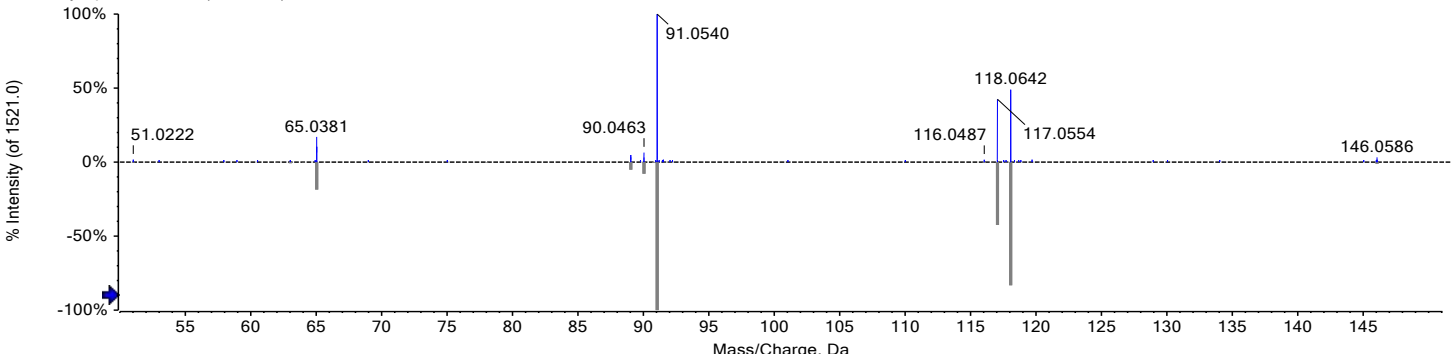

B

- Spectrum from QC-11. wiff (sample 1) - QC-11, Experiment 7, +TOF MS^2 (50 - 1000) from $1.958 \mathrm{~min}$

Precursor: $169.0 \mathrm{Da}, \mathrm{CE}: 35.0 \mathrm{CE}=35$

O Library Spectrum: Uric acid (69-93-2) , CE $=35 \pm 15$

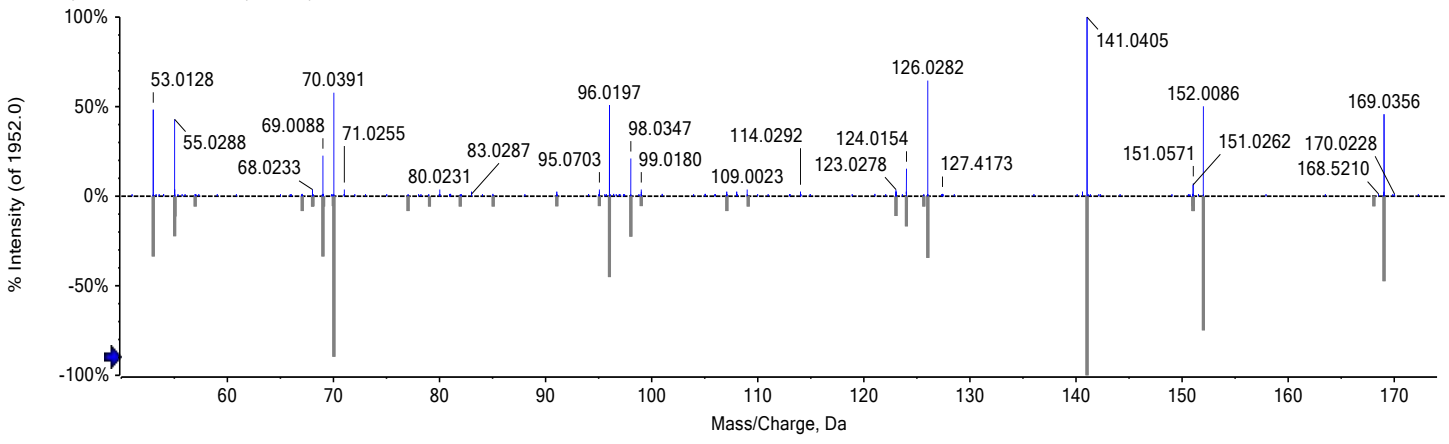

$\mathrm{C}$

- Spectrum from QC-11. wiff (sample 1) - QC-11, Experiment 4, +TOF MS^2 (50 - 1000) from $10.599 \mathrm{~min}$

Precursor: $243.1 \mathrm{Da}, \mathrm{CE}: 35.0 \mathrm{CE}=35$

O Library Spectrum: Lumichrome (1086-80-2), CE $=35 \pm 15$

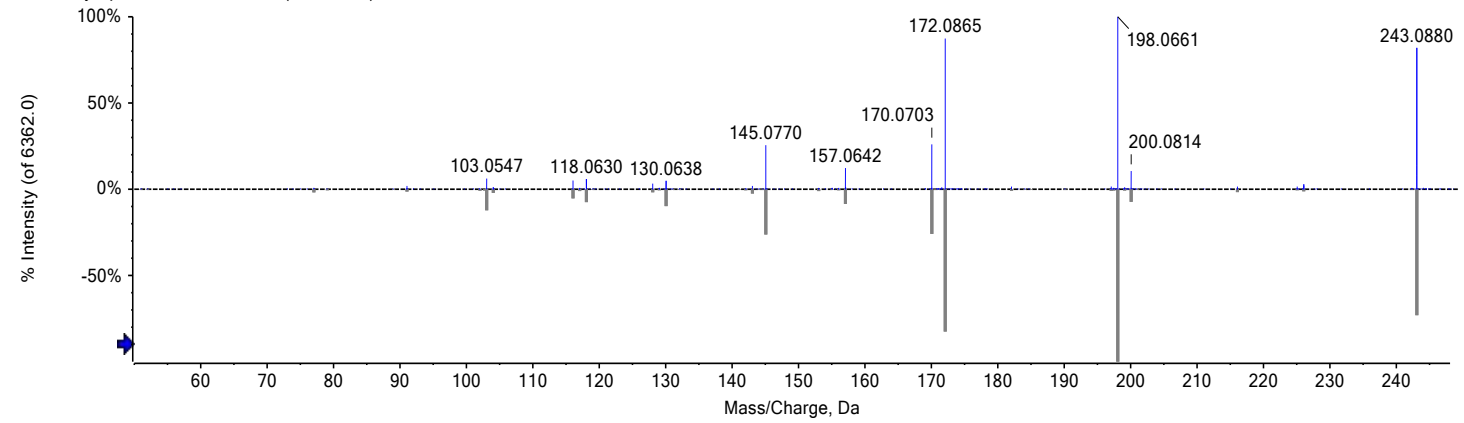


$\mathrm{D}$

- Spectrum from QC-11. wiff (sample 1) - QC-11, Experiment 7, +TOF MS^2 $(50$ - 1000) from 9.057 min Precursor: $377.1 \mathrm{Da}, \mathrm{CE}: 35.0 \mathrm{CE}=35$

O Library Spectrum: Riboflavin (83-88-5), CE $=35 \pm 15$

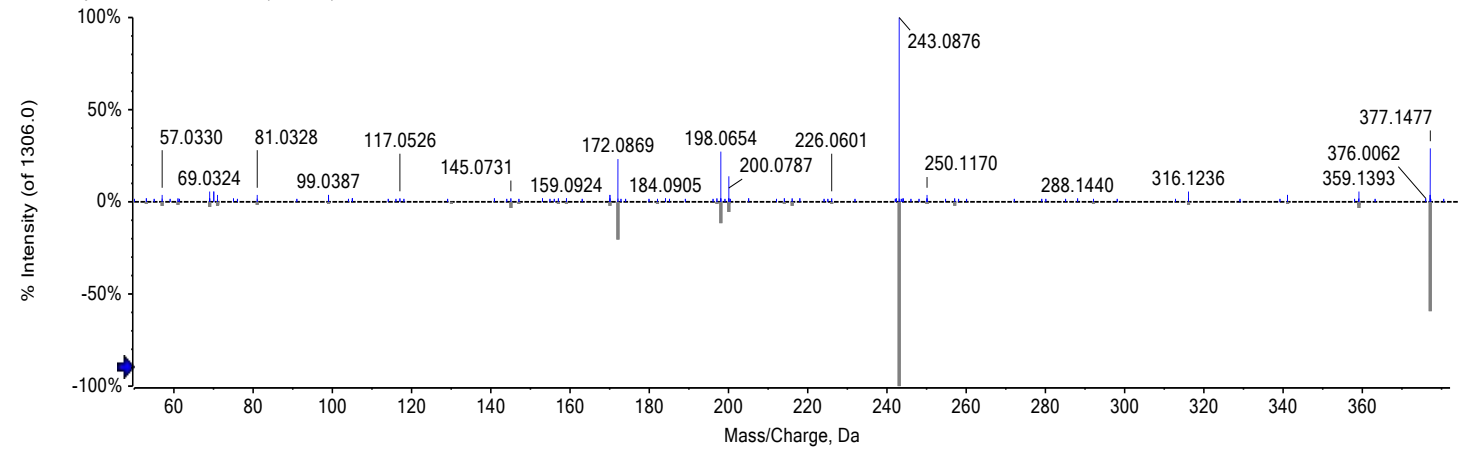

Fig. S4 MS/MS spectrums of indole-3-carboxaldehyde (A), uric acid (B), lumichrome (C) and riboflavin (D) at $35 \mathrm{eV}$ in positive ionization mode 

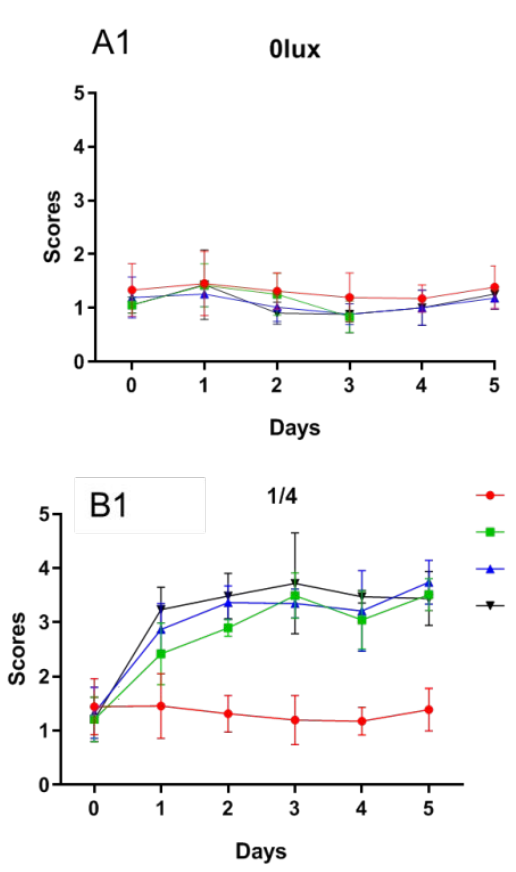

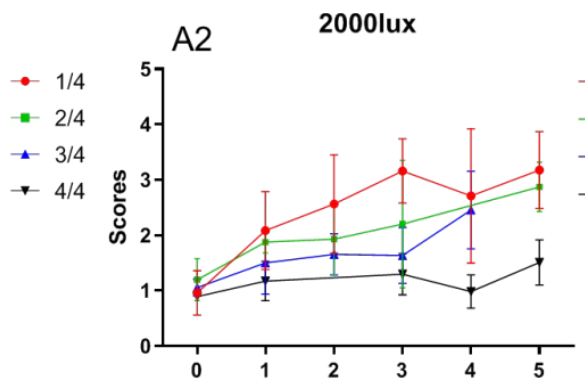

Days

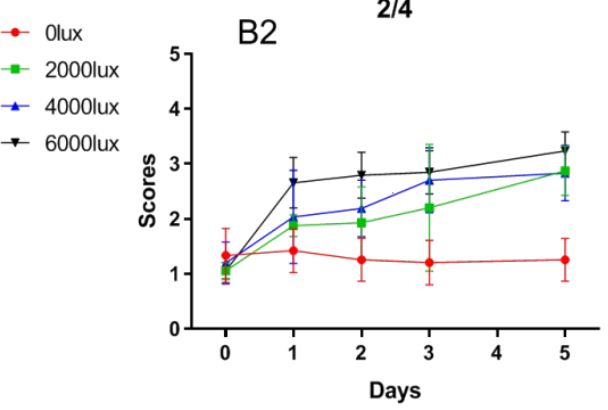

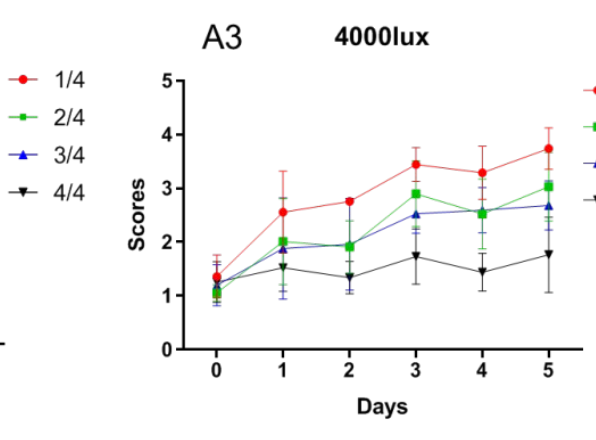

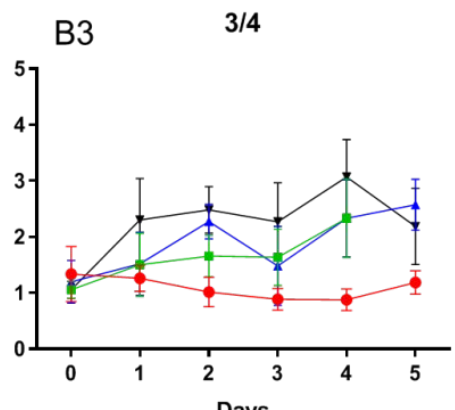

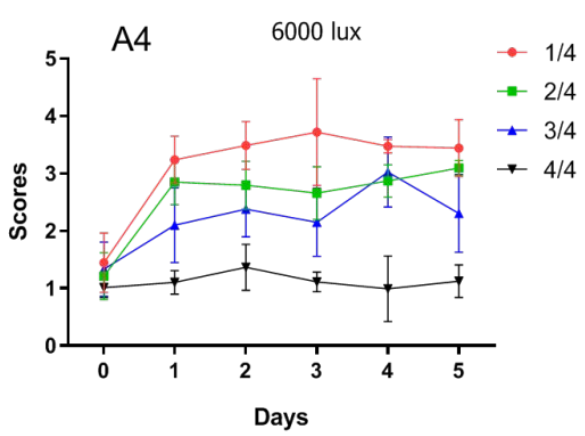

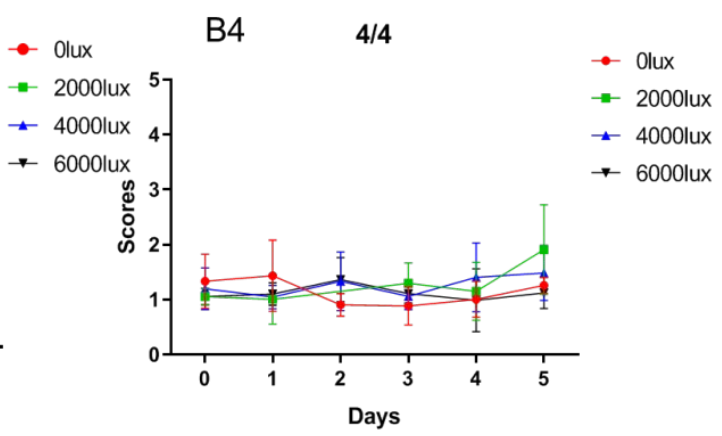

Fig. S5 Influence of sensory score by different oxygen contents and light exposure (light intensity and light duration). (A) Sensory score under different oxygen contents (1/4, 2/4, 3/4 and 4/4 of filling volume) in the presence of different light intensity ( 0 lux, 2000 lux 4000 lux and 6000 lux); (B) Sensory score under different light intensity (0, 2000, 4000 and 6000 lux $)$ in the presence of different oxygen (1/4, 2/4, 3/4 and 4/4 of filling volume) 
Table S1. The sampling information in Exp. I for identifying biomarkers

\begin{tabular}{cccccccc}
\hline $\begin{array}{l}\text { Serial } \\
\text { number }\end{array}$ & $\begin{array}{l}\text { Milk } \\
\text { brand }\end{array}$ & $\begin{array}{l}\text { Production } \\
\text { location }\end{array}$ & $\begin{array}{l}\text { Production } \\
\text { data }\end{array}$ & $\begin{array}{l}\text { Serial } \\
\text { number }\end{array}$ & $\begin{array}{l}\text { Milk } \\
\text { brand }\end{array}$ & $\begin{array}{l}\text { Production } \\
\text { location }\end{array}$ & $\begin{array}{l}\text { Production } \\
\text { data }\end{array}$ \\
\hline 1 & LQ & PL & 20200819 & 11 & DYun & AUS & 20200531 \\
2 & WDS1 & CN & 20200726 & 12 & NJ & FRA & 20200521 \\
3 & WDS2 & CN & 20200729 & 13 & NMF2 & NZL & 20200310 \\
4 & YK & UK & 20200609 & 14 & HG & NED & 20200127 \\
5 & MN & CN & 20200624 & 15 & FLM & FAR & 20200430 \\
6 & FLZ & FRA & 20200608 & 16 & AJ & NZL & 20200116 \\
7 & NMF & NZL & 20200510 & 17 & DYun2 & AUS & 20200120 \\
8 & MN & CN & 20200323 & 18 & LAJ & UK & 20191119 \\
9 & SY & CN & 20200321 & 19 & DYa & UK & 20200127 \\
10 & YL & CN & 20200819 & 20 & DY2 & UK & 20200203 \\
\hline
\end{tabular}

Table S2 The information of the potential biomarkers in Exp. I

\begin{tabular}{|c|c|c|c|c|}
\hline Precursor ion(m/z) & $\mathrm{RT}(\min )$ & VIP & $\mathrm{FC}(\mathrm{D} / \mathrm{L})$ & After lighting \\
\hline \multicolumn{5}{|c|}{ Postive } \\
\hline 377.1456 & 9.00 & 6.2 & 1254.1 & $\downarrow$ \\
\hline 169.0357 & 1.96 & 5.6 & 598.1 & $\downarrow$ \\
\hline 399.1268 & 9.03 & 6.2 & 599.6 & $\downarrow$ \\
\hline 377.1455 & 8.88 & 4.9 & 55.1 & $\downarrow$ \\
\hline 118.0647 & 10.75 & 5.6 & 0.05 & $\uparrow$ \\
\hline 146.0598 & 10.77 & 6.3 & 0.02 & $\uparrow$ \\
\hline 243.0878 & 10.61 & 4.7 & 0.12 & $\uparrow$ \\
\hline 265.0694 & 10.62 & 4.4 & 0.15 & $\uparrow$ \\
\hline 244.0905 & 10.60 & 4.4 & 0.15 & $\uparrow$ \\
\hline 485.7964 & 10.57 & 2.9 & 2.45 & $\downarrow$ \\
\hline \multicolumn{5}{|c|}{ Negative } \\
\hline 144.0456 & 10.76 & 5.2 & 0.02 & $\uparrow$ \\
\hline 241.0723 & 10.61 & 2.6 & 0.15 & $\uparrow$ \\
\hline 121.0292 & 9.64 & 3.8 & 4.7 & $\downarrow$ \\
\hline 277.0194 & 10.82 & 3.1 & 0.2 & $\uparrow$ \\
\hline
\end{tabular}


TableS3. The detailed information of sensory evaluation score by nine persons

\begin{tabular}{|c|c|c|c|c|c|c|c|c|c|c|c|}
\hline \multicolumn{3}{|c|}{ Day-filling volume-light intensity } & \multirow{2}{*}{$\frac{1 \#}{0.8}$} & \multirow{2}{*}{$\frac{2 \#}{0.0}$} & \multirow{2}{*}{$\begin{array}{l}3 \# \\
3.0\end{array}$} & \multirow{2}{*}{$\begin{array}{l}4 \# \\
1.0\end{array}$} & \multirow{2}{*}{$\begin{array}{l}5 \# \\
0.0\end{array}$} & \multirow{2}{*}{$\begin{array}{l}6 \# \\
3.8\end{array}$} & \multirow{2}{*}{$\begin{array}{l}7 \# \\
0.5\end{array}$} & \multirow{2}{*}{$\begin{array}{l}8 \# \\
0.0\end{array}$} & \multirow{2}{*}{$\begin{array}{l}9 \# \\
0.8\end{array}$} \\
\hline $1 d$ & $1 / 4$ & 0 Lux & & & & & & & & & \\
\hline $1 d$ & $2 / 4$ & 0 Lux & 0.5 & 0.0 & 2.5 & 2.5 & 0.0 & 3.0 & 0.5 & 0.0 & 1.0 \\
\hline $1 d$ & $3 / 4$ & 0 Lux & 0.3 & 0.0 & 2.5 & 2.0 & 0.0 & 3.3 & 0.6 & 0.0 & 1.0 \\
\hline $1 d$ & $4 / 4$ & 0 Lux & 1.5 & 0.0 & 2.5 & 2.5 & 0.0 & 3.4 & 0.0 & 0.0 & 1.0 \\
\hline $1 d$ & $1 / 4$ & 2000 Lux & 2.0 & 2.5 & 2.6 & 3.3 & 2.5 & 3.0 & 1.8 & 3.0 & 1.3 \\
\hline $1 d$ & $2 / 4$ & 2000 Lux & 0.8 & 1.0 & 3.0 & 2.5 & 1.5 & 4.3 & 1.3 & 2.5 & 1.0 \\
\hline $1 \mathrm{~d}$ & $3 / 4$ & 2000 Lux & 0.1 & 0.0 & 3.0 & 3.0 & 0.0 & 3.9 & 0.8 & 1.0 & 0.8 \\
\hline $1 d$ & $4 / 4$ & 2000 Lux & 0.0 & 0.0 & 3.0 & 0.5 & 0.0 & 3.6 & 0.5 & 0.0 & 0.8 \\
\hline $1 d$ & $1 / 4$ & 4000 Lux & 1.5 & 4.5 & 4.3 & 3.0 & 3.3 & 3.0 & 1.0 & 2.0 & 1.8 \\
\hline $1 d$ & $2 / 4$ & 4000 Lux & 0.3 & 1.0 & 3.5 & 1.5 & 3.5 & 3.5 & 1.0 & 1.0 & 0.9 \\
\hline $1 d$ & $3 / 4$ & 4000 Lux & 0.3 & 0.5 & 3.3 & 1.0 & 2.0 & 3.3 & 0.8 & 0.5 & 0.8 \\
\hline $1 d$ & $4 / 4$ & 4000 Lux & 0.4 & 0.0 & 2.5 & 1.0 & 0.0 & 3.5 & 0.4 & 0.5 & 1.0 \\
\hline $1 d$ & $1 / 4$ & 6000 Lux & 2.3 & 5.0 & 3.9 & 3.5 & 3.8 & 4.1 & 1.8 & 1.5 & 2.0 \\
\hline $1 d$ & $2 / 4$ & 6000 Lux & 1.3 & 4.0 & 4.3 & 3.5 & 2.5 & 3.8 & 1.5 & 1.5 & 0.8 \\
\hline $1 d$ & $3 / 4$ & $6000 \mathrm{Lux}$ & 0.2 & 1.0 & 3.8 & 3.0 & 1.5 & 3.5 & 1.3 & 0.5 & 0.8 \\
\hline $1 d$ & $4 / 4$ & 6000 Lux & 0.3 & 0.0 & 2.5 & 1.0 & 0.0 & 3.8 & 0.8 & 0.0 & 0.8 \\
\hline $2 d$ & $1 / 4$ & 0 Lux & 0.7 & 0.0 & 2.8 & 2.3 & 0.0 & 3.8 & 0.3 & 0.0 & 0.8 \\
\hline $2 d$ & $2 / 4$ & 0 Lux & 0.5 & 0.0 & 2.5 & 2.5 & 0.0 & 3.3 & 0.3 & 0.0 & 0.6 \\
\hline $2 d$ & $3 / 4$ & 0 Lux & 0.4 & 0.0 & 2.0 & 1.5 & 0.0 & 3.0 & 0.4 & 0.0 & 0.6 \\
\hline $2 d$ & $4 / 4$ & $0 \operatorname{Lux}$ & 0.2 & 0.0 & 2.0 & 1.0 & 0.0 & 3.3 & 0.3 & 0.0 & 0.6 \\
\hline $2 d$ & $1 / 4$ & 2000 Lux & 1.8 & 3.0 & 3.9 & 4.0 & 3.0 & 3.3 & 0.4 & 3.5 & 2.0 \\
\hline $2 d$ & $2 / 4$ & 2000 Lux & 0.8 & 0.5 & 2.5 & 2.5 & 3.0 & 4.0 & 0.4 & 3.5 & 1.9 \\
\hline $2 d$ & $3 / 4$ & 2000 Lux & 0.5 & 0.5 & 3.0 & 3.0 & 0.5 & 3.8 & 0.4 & 2.5 & 1.0 \\
\hline $2 d$ & $4 / 4$ & 2000 Lux & 3.8 & 0.0 & 0.5 & 0.4 & 0.0 & 3.8 & 0.4 & 0.5 & 0.5 \\
\hline
\end{tabular}




\begin{tabular}{|c|c|c|}
\hline $2 d$ & $1 / 4$ & 4000 Lux \\
\hline $2 d$ & $2 / 4$ & 4000 Lux \\
\hline $2 d$ & $3 / 4$ & 4000 Lux \\
\hline $2 d$ & $4 / 4$ & 4000 Lux \\
\hline $2 d$ & $1 / 4$ & 6000 Lux \\
\hline $2 d$ & $2 / 4$ & 6000 Lux \\
\hline $2 d$ & $3 / 4$ & 6000 Lux \\
\hline $2 d$ & $4 / 4$ & 6000 Lux \\
\hline $3 d$ & $1 / 4$ & 0 Lux \\
\hline $3 d$ & $2 / 4$ & 0 Lux \\
\hline $3 d$ & $3 / 4$ & 0 Lux \\
\hline $3 d$ & $4 / 4$ & 0 Lux \\
\hline $3 d$ & $1 / 4$ & 2000 Lux \\
\hline $3 d$ & $2 / 4$ & 2000 Lux \\
\hline $3 d$ & $3 / 4$ & 2000 Lux \\
\hline $3 d$ & $4 / 4$ & 2000 Lux \\
\hline $3 d$ & $1 / 4$ & 4000 Lux \\
\hline $3 d$ & $2 / 4$ & 4000 Lux \\
\hline $3 d$ & $3 / 4$ & 4000 Lux \\
\hline $3 d$ & $4 / 4$ & 4000 Lux \\
\hline $3 d$ & $1 / 4$ & 6000 Lux \\
\hline $3 d$ & $2 / 4$ & 6000 Lux \\
\hline $3 d$ & $3 / 4$ & 6000 Lux \\
\hline $3 d$ & $4 / 4$ & 6000 Lux \\
\hline $4 d$ & $1 / 4$ & 0 Lux \\
\hline $4 d$ & $3 / 4$ & 0 Lux \\
\hline
\end{tabular}

\begin{tabular}{|c|c|c|c|c|c|c|c|}
\hline 2.5 & 5.0 & 4.4 & 3.8 & 3.3 & 3.8 & 0.4 & 3.0 \\
\hline 0.6 & 3.5 & 4.0 & 4.0 & 2.5 & 3.0 & 0.4 & 2.5 \\
\hline 1.0 & 2.5 & 3.5 & 3.5 & 2.0 & 3.5 & 0.4 & 2.5 \\
\hline 0.6 & 0.0 & 2.5 & 3.0 & 0.0 & 3.0 & 0.3 & 0.0 \\
\hline 3.0 & 4.5 & 4.5 & 4.0 & 3.0 & 3.8 & 0.8 & 4.0 \\
\hline 1.8 & 4.0 & 4.0 & 3.0 & 2.0 & 3.8 & 0.7 & 3.5 \\
\hline 1.0 & 2.5 & 3.8 & 3.5 & 1.0 & 3.8 & 0.6 & 1.5 \\
\hline 0.4 & 0.0 & 3.0 & 2.5 & 0.0 & 4.0 & 0.4 & 0.0 \\
\hline 0.4 & 0.0 & 2.0 & 2.5 & 0.5 & 3.3 & 0.3 & 1.0 \\
\hline 0.2 & 0.0 & 2.0 & 0.5 & 0.0 & 3.5 & 0.6 & 0.0 \\
\hline 0.1 & 0.0 & 2.0 & 1.0 & 0.0 & 0.1 & 1.0 & 0.0 \\
\hline 0.4 & 0.0 & 2.0 & 0.5 & 0.0 & 3.3 & 0.4 & 0.5 \\
\hline 3.0 & 5.0 & 4.5 & 4.5 & 3.0 & 4.0 & 0.6 & 2.0 \\
\hline 2.0 & 3.0 & 1.5 & 4.0 & 1.5 & 3.8 & 0.5 & 1.0 \\
\hline 1.0 & 0.0 & 2.5 & 3.0 & 1.5 & 4.0 & 0.6 & 1.0 \\
\hline 0.5 & 0.0 & 2.5 & 1.5 & 1.5 & 3.8 & 0.8 & 0.0 \\
\hline 2.0 & 5.0 & 4.5 & 4.0 & 3.5 & 3.0 & 0.6 & 4.0 \\
\hline 0.8 & 0.5 & 3.5 & 3.0 & 2.5 & 3.9 & 0.8 & 2.5 \\
\hline 1.0 & 1.0 & 3.5 & 0.5 & 0.0 & 4.0 & 1.0 & 3.0 \\
\hline 0.3 & 0.5 & 2.5 & 1.0 & 0.0 & 0.0 & 0.0 & 0.0 \\
\hline 4.3 & 5.0 & 4.5 & 3.8 & 3.0 & 3.8 & 1.0 & 4.5 \\
\hline 1.0 & 4.0 & 3.5 & 3.5 & 2.5 & 3.8 & 1.8 & 3.5 \\
\hline 0.8 & 0.0 & 3.5 & 3.0 & 2.5 & 0.0 & 1.4 & 3.5 \\
\hline 0.4 & 0.0 & 2.5 & 1.5 & 0.0 & 4.3 & 0.4 & 0.0 \\
\hline 0.7 & 0.0 & 2.5 & 1.0 & 0.3 & 4.0 & 0.4 & 0.0 \\
\hline 0.0 & 0.0 & 2.0 & 2.0 & 0.0 & 4.2 & 0.4 & 0.0 \\
\hline
\end{tabular}




\begin{tabular}{|c|c|c|c|c|c|c|c|c|c|c|c|}
\hline $4 d$ & $4 / 4$ & 0 Lux & 4.0 & 0.0 & 0.8 & 0.4 & 0.0 & 4.0 & 0.4 & 0.0 & 0.8 \\
\hline $4 d$ & $1 / 4$ & 2000 Lux & 1.4 & 4.0 & 4.0 & 5.0 & 3.5 & 4.0 & 1.3 & 4.5 & 2.0 \\
\hline $4 d$ & $3 / 4$ & 2000 Lux & 0.8 & 0.5 & 3.5 & 3.0 & 3.5 & 4.0 & 0.6 & 2.0 & 1.8 \\
\hline $4 d$ & $4 / 4$ & 2000 Lux & 0.3 & 0.0 & 2.5 & 0.5 & 0.0 & 4.2 & 0.4 & 1.0 & 1.1 \\
\hline $4 d$ & $1 / 4$ & $4000 \mathrm{Lux}$ & 3.0 & 4.0 & 4.5 & 3.5 & 4.0 & 4.2 & 1.0 & 4.0 & 2.0 \\
\hline $4 d$ & $3 / 4$ & 4000 Lux & 1.3 & 3.0 & 4.0 & 2.5 & 3.0 & 4.2 & 1.0 & 2.5 & 1.7 \\
\hline $4 d$ & $4 / 4$ & 4000 Lux & 1.5 & 0.0 & 3.0 & 1.5 & 0.0 & 4.2 & 0.6 & 0.0 & 0.9 \\
\hline $4 d$ & $1 / 4$ & 6000 Lux & 2.3 & 4.5 & 4.5 & 4.8 & 3.8 & 4.2 & 1.8 & 5.0 & 2.0 \\
\hline $4 d$ & $3 / 4$ & 6000 Lux & 1.0 & 3.0 & 4.5 & 4.0 & 4.0 & 4.2 & 0.8 & 2.0 & 1.7 \\
\hline $4 d$ & $4 / 4$ & 6000 Lux & 0.3 & 0.0 & 3.0 & 0.0 & 0.0 & 4.2 & 0.5 & 0.5 & 1.0 \\
\hline $5 d$ & $1 / 4$ & 0 Lux & 1.0 & 0.0 & 2.3 & 2.3 & 0.5 & 3.8 & 1.3 & 4.0 & 2.0 \\
\hline $5 \mathrm{~d}$ & $2 / 4$ & 0 Lux & 3.5 & 2.5 & 1.8 & 1.3 & 2.5 & 3.5 & 1.3 & 3.0 & 1.8 \\
\hline $5 d$ & $3 / 4$ & 0 Lux & 0.4 & 0.0 & 2.5 & 1.0 & 0.5 & 3.3 & 0.4 & 4.0 & 1.3 \\
\hline $5 \mathrm{~d}$ & $4 / 4$ & 0 Lux & 0.3 & 0.0 & 2.5 & 2.0 & 0.5 & 0.3 & 2.0 & 0.5 & 2.5 \\
\hline $5 d$ & $1 / 4$ & 2000 Lux & 2.8 & 4.5 & 4.5 & 4.0 & 4.3 & 3.9 & 0.4 & 0.0 & 0.6 \\
\hline $5 d$ & $2 / 4$ & 2000 Lux & 1.5 & 3.0 & 4.5 & 3.0 & 3.0 & 3.8 & 0.4 & 0.0 & 0.8 \\
\hline $5 \mathrm{~d}$ & $3 / 4$ & 2000 Lux & 3.7 & 0.0 & 0.6 & 0.4 & 0.0 & 3.7 & 0.4 & 0.0 & 0.6 \\
\hline $5 d$ & $4 / 4$ & 2000 Lux & 0.4 & 0.0 & 3.5 & 2.5 & 3.5 & 3.5 & 0.4 & 0.0 & 0.5 \\
\hline $5 \mathrm{~d}$ & $1 / 4$ & 4000 Lux & 3.4 & 5.0 & 4.5 & 4.8 & 3.5 & 3.3 & 1.0 & 5.0 & 2.0 \\
\hline $5 \mathrm{~d}$ & $2 / 4$ & 4000 Lux & 3.0 & 4.0 & 2.0 & 0.8 & 2.0 & 3.0 & 0.8 & 4.0 & 2.0 \\
\hline $5 d$ & $3 / 4$ & 4000 Lux & 1.0 & 4.0 & 3.5 & 3.5 & 2.0 & 3.1 & 0.6 & 3.0 & 1.5 \\
\hline $5 \mathrm{~d}$ & $4 / 4$ & 4000 Lux & 0.3 & 0.0 & 3.0 & 3.0 & 0.0 & 3.0 & 0.6 & 1.0 & 1.0 \\
\hline $5 d$ & $1 / 4$ & 6000 Lux & 1.5 & 5.0 & 4.5 & 5.0 & 3.5 & 3.5 & 1.5 & 4.5 & 2.0 \\
\hline $5 d$ & $2 / 4$ & 6000 Lux & 2.0 & 4.0 & 4.0 & 5.0 & 3.0 & 4.0 & 1.0 & 4.0 & 1.9 \\
\hline $5 \mathrm{~d}$ & $3 / 4$ & $6000 \mathrm{Lux}$ & 1.8 & 0.5 & 3.5 & 4.0 & 2.5 & 3.8 & 0.6 & 2.5 & 1.9 \\
\hline $5 \mathrm{~d}$ & $4 / 4$ & 6000 Lux & 0.1 & 0.0 & 2.5 & 1.0 & 1.0 & 3.5 & 0.3 & 0.0 & 0.9 \\
\hline
\end{tabular}




\section{Targeted metabolomics analysis for tryptophan and its metabolites in milk.}

$5 \mathrm{~mL}$ of a liquid milk sample was added to a centrifuge tube, and $10 \mathrm{~mL}$ of ACN containing $5 \%$ formic acid was added. The mixture was stirred for $10 \mathrm{~min}$ at room temperature, and $1 \mathrm{~g}$ of $\mathrm{NaCl}$ and $4 \mathrm{~g}$ of $\mathrm{Na}_{2} \mathrm{SO}_{4}$ were added to promote salt-assisted liquid-liquid extraction, which was followed by stirring for $2 \mathrm{~min}$ and centrifugation at $10,000 \times \mathrm{g}$ for $10 \mathrm{~min}$. Seven milliliters of the upper layer was transferred to another centrifuge tube, and a dispersive solid-phase extraction (150 mg of C18 $+900 \mathrm{mg}$ of $\mathrm{Na}_{2} \mathrm{SO}_{4}$ ) was added. After stirring for $2 \mathrm{~min}$ and centrifugation at $10,000 \times \mathrm{g}$ for 10 min, $6 \mathrm{~mL}$ of supernatant was taken and evaporated until dry under nitrogen protection and reconstituted with $1 \mathrm{~mL}$ of $\mathrm{ACN}: \mathrm{H}_{2} \mathrm{O}(10: 90, \mathrm{v} / \mathrm{v})$ for the liquid chromatography-tandem mass spectrometry (LC-MS/MS) analysis. 